\title{
SMART KITCHEN SYSTEM USING IOT
}

\author{
Mrs Varsha R. Palandurkar, Simran J. Mascarenhas, Naaz D. Nadaf, Rupa A. Kunwar \\ Department of IT \\ AISSMS's Polytechnic, Pune, Maharashtra, India
}

\begin{abstract}
In our every day's life, safety and time is a major issue. LPG is highly flammable and if not used carefully they can be dangerous and can be sufficient to cause death. Even fire accidents occur due to leakage of gas. LPG is an odourless gas, as a result leakage detection of this gas cannot be detected by a person via smell. LPG leakage detection and location is one of the major concerns of homeowner all over the world. The idea behind our project is to give a solution that if there is gas leakage an alert message (via mail) will go to the authorized user. If due to LPG leakage, short circuit happens and causes fire, a sounding alarm will be activated and automatically the main power supply will be off. When the level of gas goes below the normal weight of cylinder, an alert message (via mail) will go to the authorized user to give a remainder for booking of gas. Also we will continuously analyze and examine the humidity and temperature of the surrounding.
\end{abstract}

Keywords - Arduino UNO, Gas sensor, Flame sensor, Humidity and Temperature sensor, Buzzer sensor, Load cell.

\section{INTRODUCTION}

Although much of the work has been done until today to realize the Internet of Things (IoT) into practice, The Internet of things (IOTs) is a network of physical objects or things embedded with electronic, software, sensors and connectivity to enable objects to exchange data with manufacturer, operators and connected devices [1]. Our project is based on IOT that is Smart Kitchen using IOT. According to news: from 2013 to 2017, the BFP recorded a total of 77,724 fire incidents, or an average of 15,545 fire incidents every year or 42 fire incidents a day. It was discovered by investigation that LPG is one of the major cause of fire [2].

The project entitled "Smart Kitchen System using IOT" will be a great help to the people in their day to day life in terms of preventing any danger caused by gas leakage [3]. The purpose of this project is to detect gas as a part of the safety system. If there is LPG detection an alert (mail) will inform the authorized user about the gas leakage in order to prevent any harmful effects due to gas leakage. We used gas sensor to monitor and detect the gas.
If due to LPG leakage, short circuit happens and causes fire, a sounding alarm will be activated and automatically the main power supply will be off. We used flame sensor for fire detection and buzzer as a sounding alarm. When the level of gas goes below the normal weight of cylinder, an alert message (via mail) will go to the authorized user to give a remainder for booking of gas. We used load cell to measure the weight of the gas cylinder. Also we will continuously analyze and examine the humidity and temperature of the surrounding. For this we used DHT11 humidity and temperature sensor to monitor humidity and temperature [4].

\section{A. General Objective}

To layout and obtain a project "Smart Kitchen using IOT".

\section{B. Specific Objective}

To layout and obtain the project that will perceive gas outflow like LPG, Butane, Methane, Alcohol, Hydrogen, Smoke that can be detected using MQ5 sensor.

To layout and setup a message alert via mail to the user.

To layout and obtain a project that will invent a sounding alarm during fire detection and the main power supply will be off.

To layout and obtain a project a project that will measure the weight of the gas and also examine the humidity and temperature.

C. Problem Statement

1. To detect the leakage of LPG system.

2. By sending alert message via mail, light control system (LED), buzzer to alert user about gas leakage [5].

\section{PROPOSED METHODOLOGY}

The purpose of this project is to detect gas as a part of the safety system. If there is LPG detection an alert (mail) will inform the authorized user about the gas leakage in order to prevent any harmful effects due to gas leakage. We used gas sensor to monitor and detect the gas.

If due to LPG leakage, short circuit happens and causes fire, a sounding alarm will be activated and automatically the main power supply will be off. We used flame sensor for fire detection and buzzer as a sounding alarm. When the level of gas goes below the normal weight of cylinder, an alert message (via mail) will go to the authorized user 
to give a remainder for booking of gas. We used load cell to measure the weight of the gas cylinder. Also we will continuously analyze and examine the humidity and temperature of the surrounding. For this we used DHT11 humidity and temperature sensor to monitor humidity and temperature.

For coding part we have used the Arduino UNO, in which we have used the Arduino Compiler (IDE) to do the coding part in order to store the values of the sensor used. Another coding part is done using Python, by using the Python Software (IDE), in which coding related to mail sending (To the user) is written [6].

\section{System Design for Smart Kitchen System using IOT}

This proposed project consists of gas leakage detection system, weight measurement module, microcontroller and alert system.

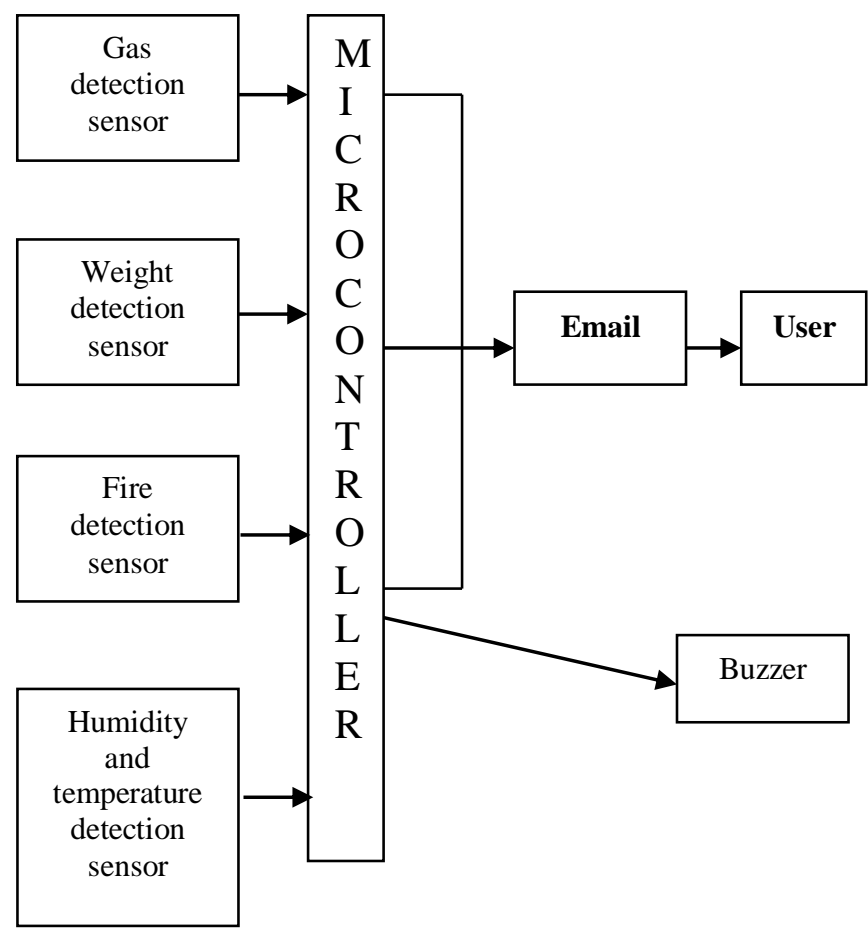

Fig 1: Smart Kitchen System using IOT

\section{SYSTEM REQUIREMENTS}

(Hardware and Software)

Hardware requirements are Arduino UNO, MQ5 sensor, DHT11 sensor, Flame sensor, Buzzer, Load cell (Weight sensor), LED, PC, Intel Core I7, 8GB RAM. Software Requirements are ARDUINO Compiler (IDE), the Python Software (IDE).

\section{HARDWARE DESCRIPTION}

\section{Arduino UNO (Microcontroller)}

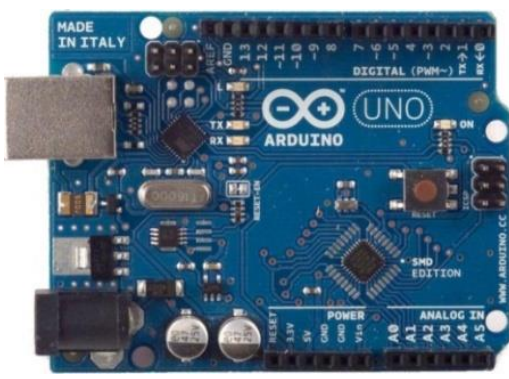

Fig 2: Arduino UNO

The Arduino Uno is a microcontroller board based on a removable, dual-inline-package (DIP) ATmega328 AVR microcontroller. It has 20 digital input/output pins (of which 6 can be used as PWM outputs and 6 can be used as analog inputs). Programs can be loaded on to it from the easy-to-use Arduino computer program.

\begin{tabular}{|c|c|c|}
\hline $\begin{array}{c}\text { Sr } \\
\text { no. }\end{array}$ & $\begin{array}{c}\text { Gas } \\
\text { Sensors }\end{array}$ & \\
\hline 1. & MQ2 & Methane, Propane, LPG, Smoke \\
\hline 2. & MQ3 & Alcohol, Grain Alcohol, Smoke \\
\hline 3. & MQ4 & Methane, Fuel and Alkane series \\
\hline 4. & MQ5 & H2, LPG, CH4, CO, Alcohol \\
\hline 5. & MQ6 & ISO-butane, Fuel and LNG \\
\hline 6. & MQ7 & Carbon monoxide \\
\hline 7. & MQ8 & Hydrogen \\
\hline 8. & MQ9 & LPG, CO, CH4 \\
\hline
\end{tabular}

Table 1: Different Types of Gas Sensor

\section{MQ5 Gas Sensor}

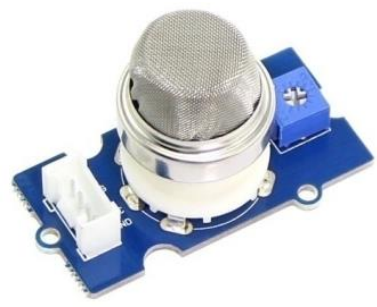

Fig 3: MQ5 Gas sensor

The Gas Sensor (MQ5) module is useful for gas leakage detection (in home and industry). It is suitable for detecting H2, LPG, CH4, CO, Alcohol. Due to its high sensitivity and fast response time, measurements can be taken as soon as possible. 


\section{Flame Sensor}

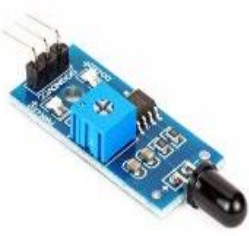

Fig 4: Flame sensor

A flame-sensor is one kind of detector which is mainly designed for detecting as well as responding to the occurrence of a fire or flame. The response of these sensors is faster as well as more accurate. This sensor uses the infrared flame flash method, which allows the sensor to work through a coating of oil, dust, water vapor, ice.

\section{DHT11 Humidity and Temperature Sensor}

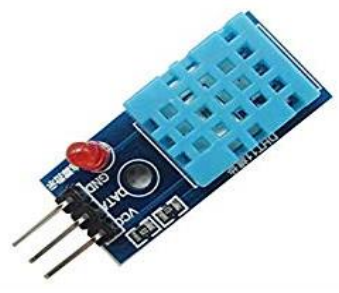

Fig 5: DHT11 Temperature and Humidity sensor DHT11 is a low-cost digital sensor for sensing temperature and humidity. This sensor can be easily interfaced with any micro-controller such as Arduino, Raspberry Pi etc., to measure humidity and temperature instantaneously. DHT11 is a relative humidity sensor.

\section{Buzzer Sensor}

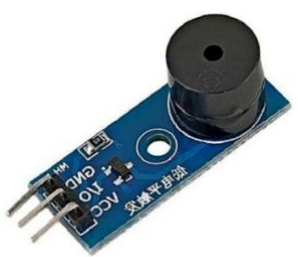

Fig 6: Buzzer sensor

The active buzzer makes sounds when triggered. It operates with 5VDC. You can use any of the Arduino IOs to control this module. It makes sounds when IO connected is HIGH. You can remove the label on the top to make the sounds louder.

\section{Load cell (Weight Sensor)}

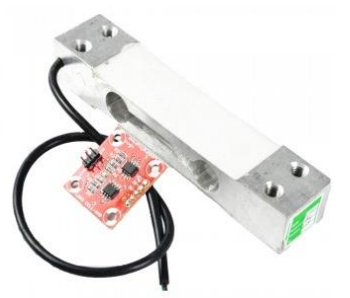

Fig 7: Load cell

A load cell is a type of transducer, specifically a force transducer. It converts a force such as tension, compression, pressure, or torque into an electrical signal that can be measured and standardized. As the force applied to the load cell increases, the electrical signal changes proportionally.

\section{LED}

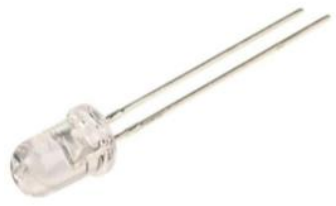

Fig 8: LED

LED is the acronym for "Light Emitting Diode". LEDs are semiconductor devices that produce light. These were initially used as indicator lights but are now used extensively for indoor and outdoor lighting.

\begin{tabular}{|c|l|c|c|}
\hline $\begin{array}{c}\text { Sr } \\
\text { no. }\end{array}$ & \multicolumn{1}{|c|}{ Test Condition } & LED & Buzzer \\
\hline 1. & Gas Leakage & Off & On \\
\hline 2. & No Gas Leakage & Glow & Off \\
\hline
\end{tabular}

Table 2: Test Condition for LED and Buzzer

\section{SOFTWARE DESCRIPTION}

\section{The Arduino IDE}

The Arduino IDE is a cross-program application created in Java and is originate from IDE for the deal with a programming language and the wiring project. It is proposed to establish programming to a performer and other new users unfamiliar with software enhancement. It includes code editor with features such us syntax importance, automatic indentation, brace matching, and is also qualified of compiling and uploading a package to the board with a specific click [7].

\section{The Python IDLE}

IDLE (Integrated Development and Learning Environment) is an integrated development environment (IDE) for Python. IDLE has two main window types, the Shell window and the Editor window. It is possible to have multiple editor windows simultaneously. IDLE is an integrated development environment for Python, which has been bundled with the default implementation of the language since $1.5 .2 \mathrm{~b} 1$. It is packaged as an optional part of the Python packaging with many Linux distributions. It is written in Python [8]. 


\section{OPERATION OF SYSTEM}

In this system, the MQ5 gas sensor is used to sense the LPG, methane, butane and various gases. This sensor sends a signal (digital pulse) to the microcontroller when gas is being leaked. An alert message is sent through mail to the authorized user about gas leakage. The Flame sensor is used to detect fire or flame. If due to gas leakage, a short circuit takes place and causes fire, the flame sensor will sense it and send the signal to the microcontroller about the fire. An alert message is sent through mail to the authorized user, a buzzer alarm is activated and automatically the main power supply will be off. This alarm produces a huge sound which drops down the attention of the user [9], [10].

The Load cell (Weight sensor) will sense the weight of the gas cylinder and continuously send the signals to the microcontroller. When the threshold value (set) reaches below predetermined value, an alert message through mail will be sent to the user as a remainder to book the gas [11], [12].

The DHT11 sensor for humidity and temperature will continuously monitor and examine the surrounding humidity and temperature and send the signals to the microcontroller.

\section{FLOWCHART}

\section{For LPG Leakage}

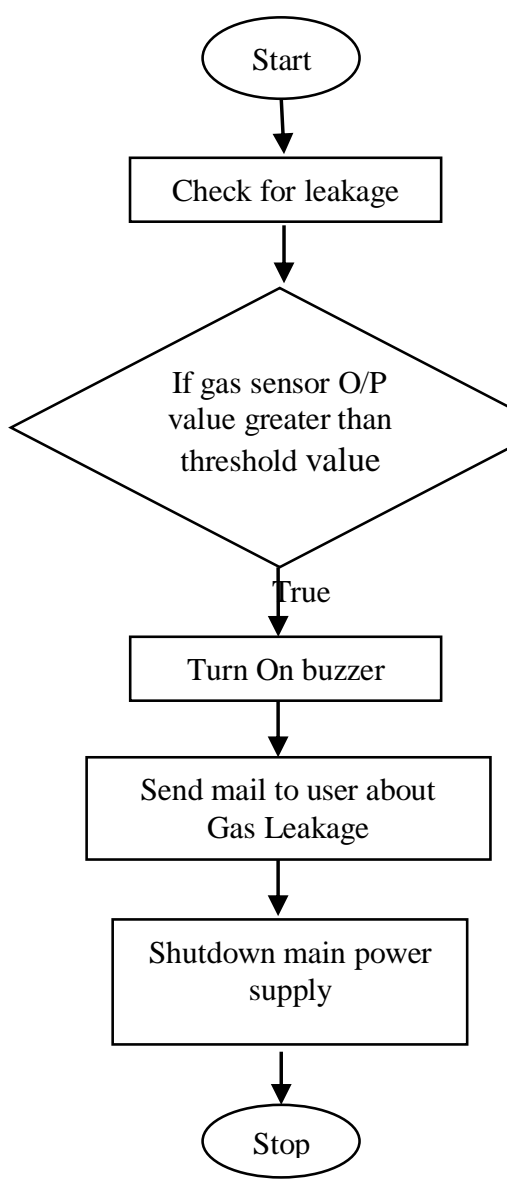

Fig 9: Flowchart for LPG Leakage

\section{For Gas Booking alert}

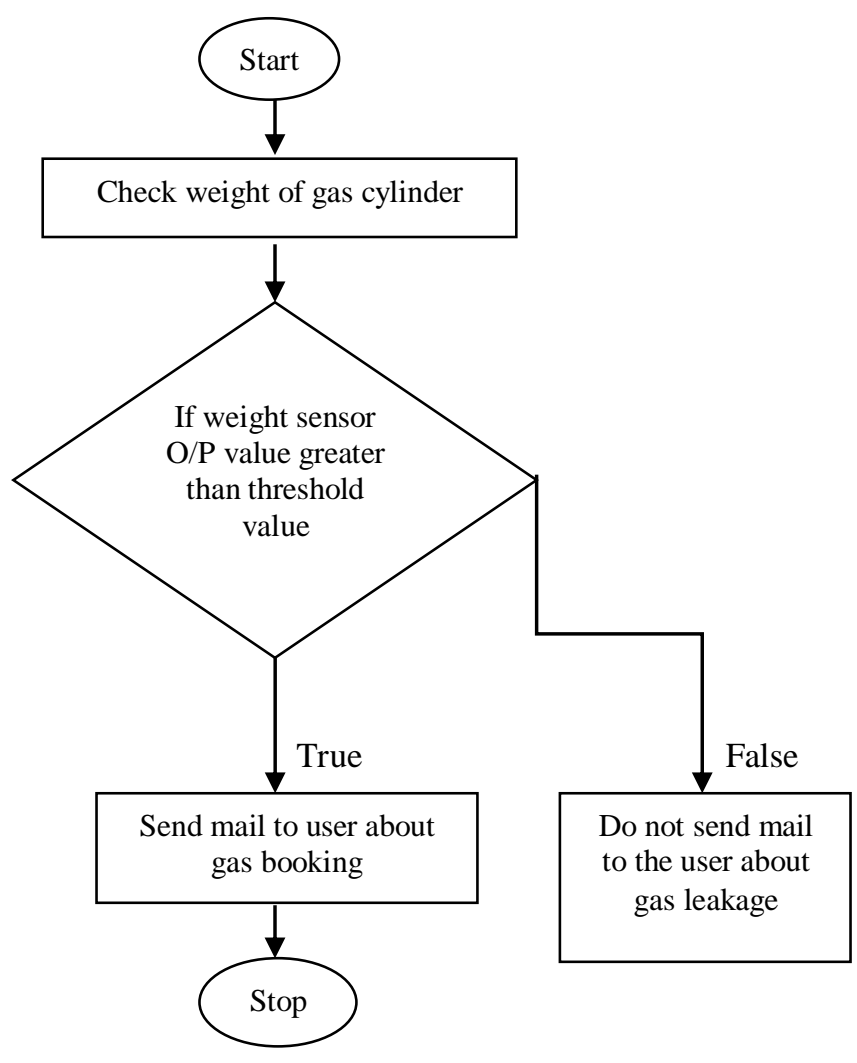

Fig 10: Flowchart for Gas Booking alert

VIII. RESULTS

\section{Hardware Implementation:}

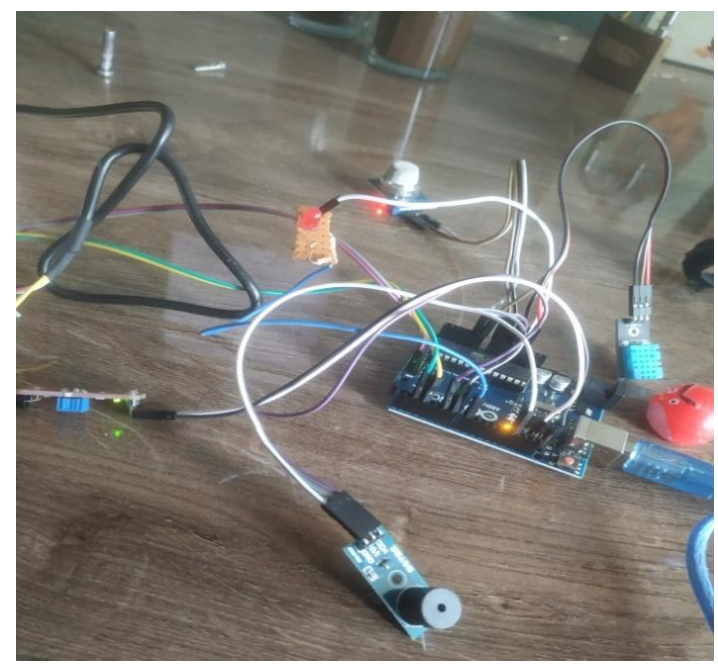

Fig 9: Overall Hardware Setup 


\section{Output for Mail when Gas is detected:}

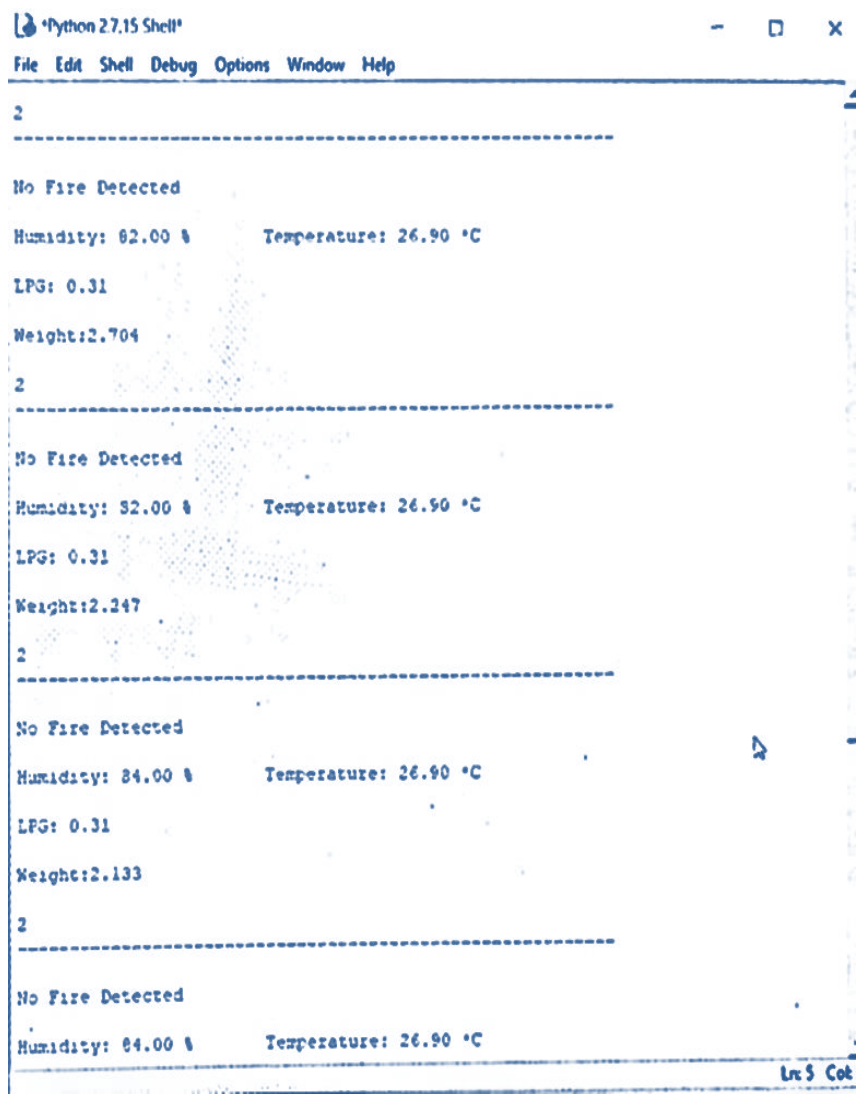

Fig 11: Message is sent

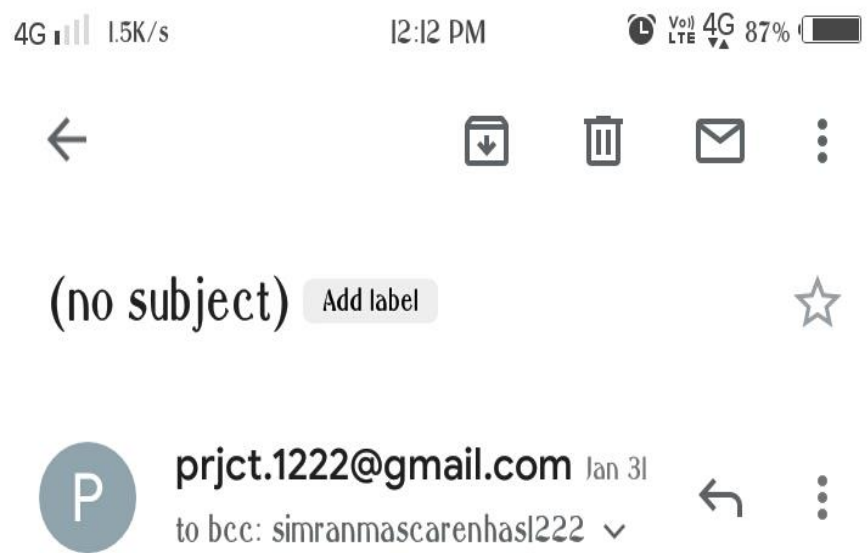

Gas booking

Fig 12: Received message to Gmail
$4 G$ II I. $3 K / s$
12:12 PM
(৫) 닌) 4 G $87 \%$ '

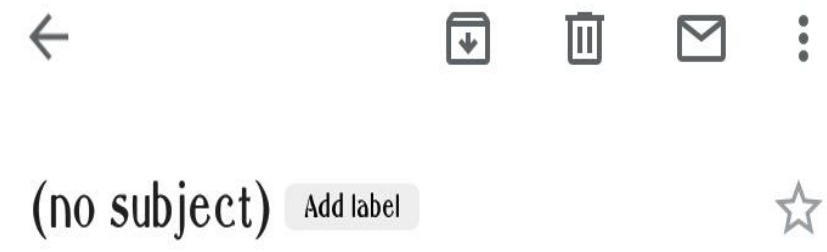

P

\section{prjct.1222@gmail.com Feb 17 \\ to bcc: simranmascarenhasl222 $\vee$

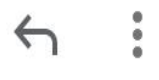

Fire detected

Fig 13: Received message to Gmail

\section{CONCLUSION}

Gas leakage leads to severe accidents resulting in material losses and human injuries. Gas leakage occurs mainly due to poor maintenance of equipments and inadequate awareness of the people. Hence, LPG leakage detection is essential to prevent accidents and to save human lives. This monitoring and detection system is proposed mainly to meet the safety standards and to avoid fire accidents because of leakage.

In this system, if there is gas leakage an alert message (via mail) will go to the authorized user. If due to LPG leakage, short circuit happens and causes fire, a sounding alarm will be activated and automatically the main power supply will be off. When the level of gas goes below the normal weight of cylinder, an alert message (via mail) will go to the authorized user to give a remainder for booking of gas. Also we will continuously analyze and examine the humidity and temperature of the surrounding. This system is very simple yet reliable.

\section{REFERENCES}

[1] S. K. K. T. K. R. R.Naresh Nuke, P.Siva Nagendra Reddy. (2016). Arduino based LPG gas monitoring, automatic cylinder booking with alert system. IOSR Journal of Electronics and Communication Engineering (IOSR-JECE), (Vol. 11, 2016).

[2] S. K. D. P. S. R. Abid Khan, Neju K. Prince. (2014). GSM based automatic LPG ordering system with leakage alert. IJRET. (Vol. 3).

[3] A. Gupta. (2017). LPG leakage detector and auto shutoff system using Arduino UNO ATmega328. International Journal of Innovative Research in Science, Engineering and Technology.(Vol. 6). 
[4] B.V.Prasanth. (2014). Cloud connected smart gas leakage detection and safety precaution system. International Journal of MC Square Scientific Research.(vol 6).

[5] S. H. Kumar Keshamoni. (2017). Smart gas level monitoring booking gas leakage detector over IOT. International Journal of MC Square Scientific Research. (Vol. 1).

[6] Tanvira Ismail. (2014). GSM Based Gas Leakage Detection System with Preventive Measures. Int. J. Elect. And Comm.Engg \& Tech. (pp. 135).

[7] R. N. Naik, P. S. N. Reddy, S. N. Kishore, and K. T. Kumar Reddy. (2016). Arduino Based LPG gas Monitoring \& Automatic Cylinder booking with Alert System. IOSR J. Electron. Commun.Eng., (vol. 11, no. 4, pp. 06-12).

[8] A. Shrivastava, R. Prabhaker, R. Kumar, and R. Verma. (2013). Gsm Based Gas Leakage Detection System. International Journal of Emerging Trends in Electrical and Electronics. (vol. 1, no. 2, pp. 42-45).

[9] S. P. S. Selvapriya C, A. M, and A. K. C. (2013). LPG Leakage Monitoring and Multilevel Alerting System. Int. J. Eng. Sci. Res. Technol. (vol. 2, no. 11, pp. 1-4).

[10] V. Abishek and M. Aierselvam. (2013). Wireless Auto Power Trip during Gas Leakage. Advance in Electronic and Electric Engineering. (vol. 3, no. 3, pp. 327-332).

.[11] Z. Yang, M. Liu, M. Shao, and Y. Ji. (2011). Research on Leakage Detection and Analysis of Leakage Point in the Gas Pipeline System. Open J. Saf. Sci. Technol. (vol. 1, no. 3, pp. 94-100).

[12] E. Kim et al. (2012). Pattern Recognition for Selective Odor Detection with Gas Sensor Arrays. Sensors. (vol. 12, no. 12, pp. 16262-16273). 